Document downloaded from:

http://hdl.handle.net/10251/78849

This paper must be cited as:

Calabuig, JM.; Jiménez Fernández, E.; Juan Blanco, MA.; Sánchez Pérez, EA. (2016). Tensor product representation of Kothe-Bochner spaces and their dual spaces. Positivity. 20(1):155-169. doi:10.1007/s11117-015-0347-3.

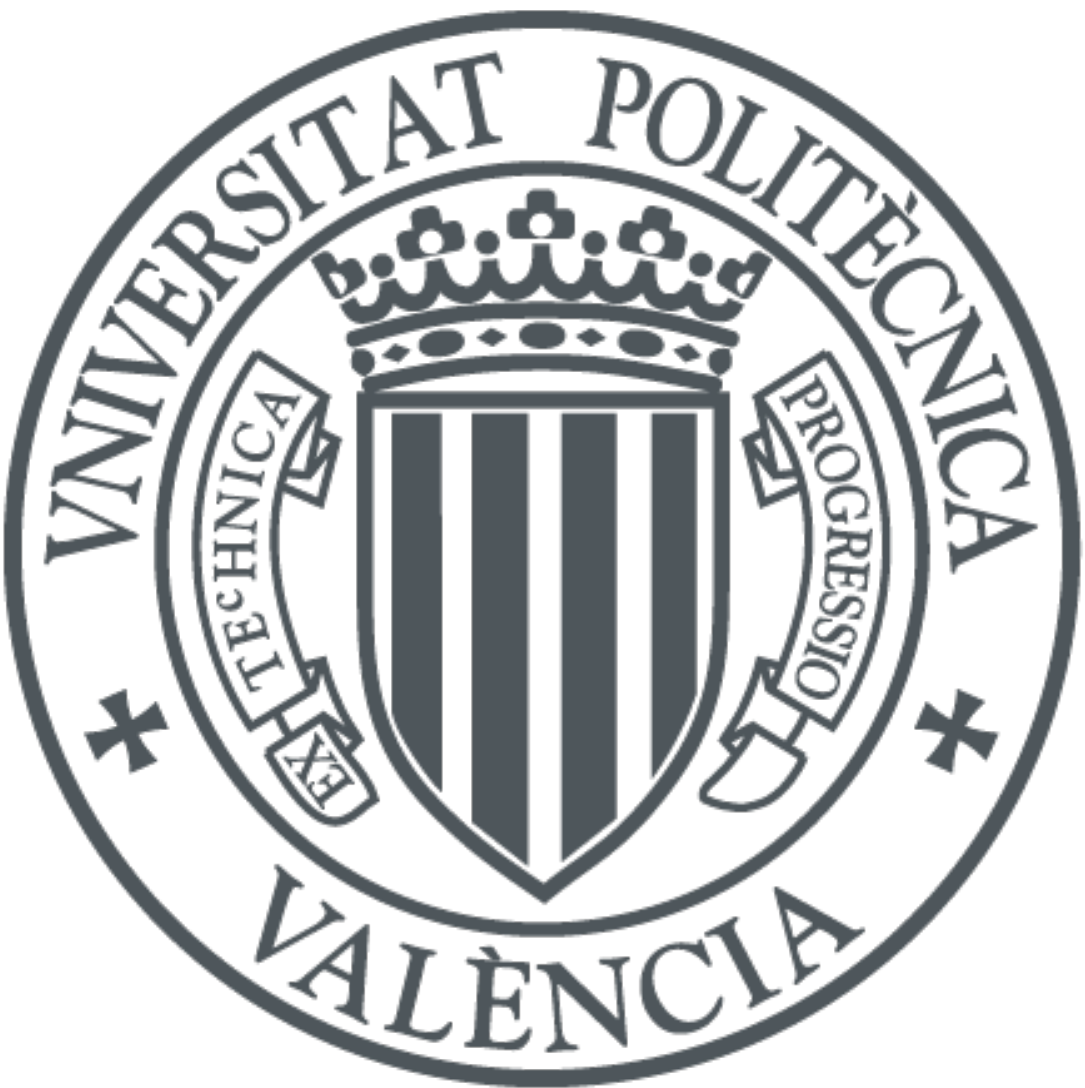

The final publication is available at

http://dx.doi. org/10.1007/s11117-015-0347-3

Copyright Springer Verlag

Additional Information 


\title{
TENSOR PRODUCT REPRESENTATION OF KÖTHE-BOCHNER SPACES AND THEIR DUAL SPACES
}

\author{
J.M. CALABUIG, E. JIMÉNEZ FERNÁNDEZ, M.A. JUAN AND E.A. SÁNCHEZ PÉREZ
}

\begin{abstract}
We provide a tensor product representation of Köthe-Bochner function spaces of vector valued integrable functions. As an application, we show that the dual space of a Köthe-Bochner function space can be understood as a space of operators satisfying a certain extension property. We apply our results in order to give an alternate representation of the dual of the Bochner spaces of $p$-integrable functions and to analyze some properties of the natural norms $\Delta_{p}$ that are defined on the associated tensor products.
\end{abstract}

\section{Introduction}

Consider a Banach function space $X(\mu)$ over a finite measure space $(\Omega, \Sigma, \mu)$ and a Banach space $Y$. The first elements for the definition of the so called KötheBochner spaces $X(\mu, Y)$ appeared for the first time in the mathematical literature some 80 years ago, with the original work of Bochner [1]. From this moment on, these spaces have been intensively studied, and some new presentation and applications have been obtained in recent years (see [16] and the references therein). On the other hand, vector valued functions that are integrable with respect to vector measures have been studied since the 50'. The so called tensor valued integration (vector valued functions that are integrable with respect to vector measures find their integrals in a topological tensor product, that is usually considered to the injective one), started with the work of Dobrakov (see [11] and the previous papers of the same author), and together with the integration of scalar functions with respect to vector measures that was developed by Lewis [15] and other authors (see [18] and the references therein) lead to a well supported tensor integration theory, whose main results and applications can be found in a series of recent papers (see $[6,7,12,14,19,20]$ and the references therein).

Following the research in this direction - the study of the spaces of vector valued functions that are integrable with respect to vector valued measures-, the aim of this paper is to extend the well-known representation of spaces of Bochner integrable

2000 Mathematics Subject Classification. Primary 46E30, Secondary 46B28, 46G10, , 46B42.

Key words and phrases. vector measure, Banach function space, tensor product, dual space .

First and third authors are supported by grant MTM2011-23164 of the Ministerio de Economía y Competitividad (Spain). Second and fourth authors are supported by grant MTM2012-36740C02-02 of the Ministerio de Economía y Competitividad (Spain). 
functions as projective tensor products. This will allow to identify dual spaces of Köthe-Bochner spaces with spaces of operators with a particular factorization property. Our technique uses the nowadays well-known representation procedure for Banach function spaces by means of spaces of integrable functions with respect to vector measure (see [18, Ch.3] and [9, 2] for more recent results). We show also some concrete representations of dual spaces of Köthe-Bochner spaces $X(\mu, Y)$ for order continuous Banach function spaces $X(\mu)$ on a finite measure $\mu$ in terms of the elements of the dual space $X(\mu)^{*}$ and some applications related with the natural tensor norms $\Delta_{p}$ on tensor products $L^{p}(\mu) \otimes Y$.

\section{BASIC DEFINITIONS AND NOTATION}

We will use standard Banach space definitions and notation. If $X$ is a Banach space, we write $B_{X}$ for its closed unit ball and $X^{*}$ for its dual space. If $L$ is a Banach lattice we will write as usual $L^{+}$for its positive cone. Let $(\Omega, \Sigma)$ be a measurable space. Let $\nu: \Sigma \rightarrow X$ be a Banach space valued vector measure. Associated to the measure $\nu$ there is a class of scalar measures defined as the variations $\left|\left\langle\nu, x^{*}\right\rangle\right|$ of all the scalarizations $\left\langle\nu, x^{*}\right\rangle(\cdot):=x^{*}(\nu(\cdot))$ of the measure $\nu, x^{*} \in B_{X^{*}}$. A Rybakov measure for $\nu$ (see [10, Chapter IX]) is a scalar measure $\left|\left\langle\nu, x^{*}\right\rangle\right|$ defined as the variation of the composition of $\nu$ with an element $x^{*} \in X^{*}$ in such a way that $\nu$ and $\left|\left\langle\nu, x^{*}\right\rangle\right|$ have the same null sets. A measurable function $f: \Omega \rightarrow \mathbb{R}$ is said to be integrable with respect to $\nu$ if it is integrable with respect to all the scalar measures $\left\langle\nu, x^{*}\right\rangle, x^{*} \in X^{*}$, and for each $A \in \Sigma$ there is a (unique) vector $\int_{A} f d \nu \in X$ such that

$$
\int_{A} f d\left\langle\nu, x^{*}\right\rangle=\left\langle\int_{A} f d \nu, x^{*}\right\rangle, \quad x^{*} \in X^{*} .
$$

The set consisting of equivalence classes of ( $\mu$-almost everywhere equal) integrable functions, where $\mu$ is a Rybakov measure for $\nu$, will be denoted by $L^{1}(m)$ and it is an order continuous Banach function space over $\mu$, when we endow it with the lattice norm

$$
\|f\|_{L^{1}(m)}=\sup \left\{\int_{\Omega}|f| d\left|\left\langle m, x^{*}\right\rangle\right|: x^{*} \in B_{X^{*}}\right\},
$$

with a weak unit given by $\chi_{\Omega}$. We follow the definition of Banach function space given in $[17$, p.26] (Köthe function space). The reader can find all the information that is needed on integration of scalar functions with respect to vector measures and the corresponding spaces of integrable functions in [18]. An important fact regarding these spaces that will be used in the paper is the following representation theorem: every order continuous Banach lattice with a weak order unit can be written isometrically and in the order as a space $L^{1}(\nu)$ of a suitable vector measure $\nu$ (Proposition 3.9 in [18]).

Let us recall now some notions on (scalar measure) integration of vector valued functions. Let $X(\mu)$ be a Banach function space and $Y$ a Banach space. The 
Köthe-Bochner space $X(\mu, Y)$ is defined to be the space of ( $\mu$-a.e. classes of) strongly measurable functions $f$-using $\mu$ as reference measure- such that the function $w \rightsquigarrow\|f(w)\|_{Y}$ belongs to $X(\mu)$, with the norm

$$
\|f\|_{X(\mu, Y)}:=\|\| f\left\|_{Y}(w)\right\|_{X(\mu)} .
$$

The so defined space $\left(X(\mu, Y),\|f\|_{X(\mu, Y)}\right)$ is a Banach space of classes of strongly measurable functions that are equal $\mu$-a.e. Some references in which the fundamental properties of these spaces can be found are $[5,13,16]$.

\section{Tensor Product Representation of KÖthe-Bochner spaces}

In this section we are interested in providing an extension of the well-known representation theorem that allows to write spaces of Bochner integrable functions as projective tensor products: if $Y$ is a Banach space and $\mu$ a finite measure, it is well-known that

$$
L^{1}(\mu, Y)=L^{1}(\mu) \hat{\otimes}_{\pi} Y,
$$

the completion of the projective tensor product (see for example Proposition 1.8.6 in [16]). The definition of norm of the space $L^{1}(\nu)$ of a vector measure $\nu$ provides a natural candidate for representing the Köthe-Bochner spaces $X(\mu, Y)$-which by the representation theorem [18, Prop.3.9] can be written as $L^{1}(\nu, Y)$ for a certain vector measure $\nu$-, as the completion of normed tensor products. For the case of positive vector measures, this representation implies also a characterization of the elements of the dual space involving factorizations of associated operators.

3.1. The representation theorem. Let $(\Omega, \Sigma)$ be a measurable space and $\nu$ : $\Sigma \rightarrow X$ a vector measure. Let $\nu: \Sigma \rightarrow X$ be a Banach space valued vector measure and $Y$ a Banach space. In what follows $\mu$ will be a scalar Rybakov measure for $\nu$. Consider the Köthe-Bochner space $L^{1}(\nu, Y)$. An important fact is that since $L^{1}(\nu)$ is order continuous, simple functions are dense in the corresponding Köthe-Bochner space with the norm defined by the expression above (see for example [13, Lema $1.51])$.

Consider the set of all the vector measures associated to $\nu$. We can define the following seminorm on the tensor product $L^{1}(\nu) \otimes Y$. If $u=\sum_{i=1}^{n} h_{i} \otimes y_{i} \in L^{1}(\nu) \otimes Y$, then we define $\alpha_{\nu}$ as

$$
\alpha_{\nu}(u):=\sup _{x^{*} \in B_{X^{*}}} \inf _{\operatorname{rep}(u)}\left(\sum_{i=1}^{n}\left\|h_{i}\right\|_{L^{1}\left(\left|\left\langle\nu, x^{*}\right\rangle\right|\right)} \cdot\left\|y_{i}\right\|_{Y}\right),
$$

where $\operatorname{rep}(u)$ denotes the set of all the representations of $u$ as the one given above.

Theorem 1. Let $\nu$ be a Banach space valued vector measure and $Y$ a Banach space. Then

$$
L^{1}(\nu, Y)=L^{1}(\nu) \hat{\otimes}_{\alpha_{\nu}} Y .
$$


Proof. Take a simple function $f \in L^{1}(\nu, Y)$. By definition of the norm, we have that

$$
\|f\|_{L^{1}(\nu, Y)}:=\sup _{x^{*} \in B_{X^{*}}} \int\|f\|(w) d\left|\left\langle\nu, x^{*}\right\rangle\right| .
$$

Consider a representation of $f:=\sum_{i=1}^{k} h_{i} \cdot y_{i}$, that is naturally associated to the tensor $u=\sum_{i=1}^{k} h_{i} \otimes y_{i}$, and take an element $x_{0}^{*} \in B_{X^{*}}$. Then

$$
\|f\|_{L^{1}\left(\left|\left\langle\nu, x_{0}^{*}\right\rangle\right|\right)} \leq \sum_{i=1}^{k} \int\left|h_{i}\right| d\left|\left\langle\nu, x_{0}^{*}\right\rangle\right| \cdot\left\|y_{i}\right\| .
$$

Then it is clear that

$$
\|f\|_{L^{1}\left(\left|\left\langle\nu, x_{0}^{*}\right\rangle\right|\right)} \leq \inf _{\operatorname{rep}(u)}\left\|h_{i}\right\|_{L^{1}\left(\left|\left\langle\nu, x_{0}^{*}\right\rangle\right|\right)} \cdot\left\|y_{i}\right\|_{Y} \cdot
$$

Consequently, by computing suprema over $x^{*} \in B_{X^{*}}$ we obtain that $\|f\|_{L^{1}(\nu, Y)} \leq$ $\alpha_{\nu}(u)$.

For the converse just use the standard representation of the simple function $f$ given by

$$
f=\sum_{i=1}^{n} y_{i} \chi_{A_{i}}
$$

where the sets $A_{i}$ are disjoint measurable sets. For each $x^{*} \in B_{X^{*}}$ we obtain that

$$
\sum_{i=1}^{n}\left|\left\langle\nu, x^{*}\right\rangle\right|\left(A_{i}\right) \cdot\left\|y_{i}\right\|=\|f\|_{L^{1}\left(\left|\left\langle\nu, x^{*}\right\rangle\right|, Y\right)},
$$

and so

$$
\inf _{\operatorname{rep}(u)}\left(\sum_{i=1}^{k}\left\|h_{i}\right\|_{L^{1}\left(\left|\left\langle\nu, x^{*}\right\rangle\right|\right)} \cdot\left\|y_{i}\right\|_{Y}\right) \leq\|f\|_{L^{1}(\nu, Y)},
$$

which gives by computing the supremum over $x^{*} \in B_{X^{*}}$ of the left hand side expression the inequality

$$
\alpha_{\nu}(u) \leq\|f\|_{L^{1}(\nu, Y)} .
$$

This proves the result for simple functions, that can be extended to all the functions of the space using the fact that simple functions are dense.

Example 2. (1) Consider a finite measure $(\Omega, \Sigma, \mu)$ space and take a disjoint infinite partition $\left\{A_{i}\right\}_{i \in \mathbb{N}}$ defined by non-null measurable sets. Let $1<p<\infty$ and define the space $Z:=\bigoplus_{i=1, p}^{\infty} L^{1}\left(\mu_{i}\right)$, the $p$-direct sum of the Lebesgue spaces $L^{1}\left(\mu_{i}\right)$, where $\mu_{i}$ is the measure $\mu$ restricted to the sets $A_{i}, i \in \mathbb{N}$. Take the vector measure $m: \Sigma \rightarrow \ell^{p}$ given by $m(A):=\sum_{i=1}^{\infty} \mu_{i}\left(A \cap A_{i}\right) e_{i}$, where $e_{i}$ is the $i$-th vector of the canonical basis of $\ell^{p}$ and $A \in \Sigma$. It can be easily proved that $Z$ can be identified with $L^{1}(m)$. Fix a norm one positive sequence $\left(\lambda_{i}\right) \in \ell^{p^{\prime}}, \lambda_{i}>0$ for all $i \in \mathbb{N}$, and consider the (positive scalar) measure $\eta(A):=\left\langle\left(\lambda_{i}\right), m\right\rangle(A)=\bigoplus_{i=1}^{\infty} \lambda_{i} \mu\left(A \cap A_{i}\right)$, $A \in \Sigma$. This is a Rybakov measure for $m$. 
Take now a Banach space $Y$ and consider the Köthe-Bochner space $Z(\eta, Y)$. In the following calculations we will write $x^{*}=\left(x_{i}^{*}\right)$ for the elements $x^{*} \in \ell^{p^{\prime}}$. Theorem 1 asserts that

$$
L^{1}(m, Y)=L^{1}(m) \hat{\otimes}_{\alpha_{m}} Y,
$$

and in this case, for a tensor $u \in L^{1}(m) \otimes Y$,

$$
\begin{aligned}
& \alpha_{m}(u):=\sup _{x^{*} \in B_{\ell p^{\prime}}} \inf _{\operatorname{rep}(u)}\left(\sum_{j=1}^{n}\left\|h_{j}\right\|_{L^{1}\left(\left|\left\langle m, x^{*}\right\rangle\right|\right)} \cdot\left\|y_{j}\right\|_{Y}\right) \\
= & \sup _{x^{*} \in B_{\ell^{p^{\prime}}} \cap\left(\ell^{p^{\prime}}\right)+\operatorname{rep}(u)} \inf _{j=1}\left(\sum_{i=1}^{n} \sum_{A_{i}}^{\infty}\left|h_{j}\right| d\left\langle m, x^{*}\right\rangle \cdot\left\|y_{j}\right\|_{Y}\right) \\
= & \sup _{x^{*} \in B_{\ell p^{\prime}} \cap\left(\ell^{p^{\prime}}\right)+} \inf _{\operatorname{rep}(u)}\left(\sum_{i=1}^{\infty} x_{i}^{*} \int_{A_{i}} \sum_{j=1}^{n}\left|h_{j}\right|\left\|y_{j}\right\|_{Y} d \mu_{i}\right),
\end{aligned}
$$

from which, using an approximation argument based on the small size of the integrals in $A_{i}$ for $i$ big enough, and taking into account that the projective tensor product of an $L^{1}$ space with a Banach space is isometric to the corresponding Bochner space, we get that $\alpha_{m}(u)$ is equal to

$$
\begin{gathered}
\sup _{x^{*} \in B_{\ell p^{\prime}} \cap\left(\ell^{p^{\prime}}\right)^{+}}\left(\sum_{i=1}^{\infty} x_{i}^{*} \inf _{\operatorname{rep}\left(u \chi_{A_{i}}\right)} \int_{A_{i}} \sum_{j=1}^{n}\left|h_{j}^{i}\right|\left\|y_{j}\right\|_{Y} d \mu_{i}\right) \\
=\sup _{x^{*} \in B_{\ell p^{\prime}} \cap\left(\ell^{p^{\prime}}\right)^{+}}\left(\sum_{i=1}^{\infty} x_{i}^{*}\left\|\sum_{j=1}^{n} \chi_{A_{i}} h_{j} \cdot y_{j}\right\|_{L^{1}\left(\mu_{i}, Y\right)}\right) \\
=\left(\sum_{i=1}^{\infty}\left\|\left(\sum_{j=1}^{n} h_{j} \cdot y_{j}\right) \chi_{A_{i}}\right\|_{L^{1}\left(\mu_{i}, Y\right)}^{p}\right)^{1 / p} .
\end{gathered}
$$

That is, the Köthe-Bochner space $Z(\eta, Y)$ coincides isometrically with the disjoint $p$-sum of Bochner spaces $\bigoplus_{i=1, p}^{\infty} L^{1}\left(\mu_{i}, Y\right)$, and the norm coincide with $\alpha_{m}$.

(2) The same computations gives the general case involving disjoint sums with respect to Orlicz sequence norms. Let $\phi$ be an order continuous Orlicz norm, and with the same construction that in (1), consider the Banach function space $Z_{\phi}:=\bigoplus_{i=1, \phi}^{\infty}$ and the vector measure $m: \Sigma \rightarrow \ell^{\phi}$. The corresponding norm $\alpha_{m}^{\phi}$ is given in this case by the expression

$$
\alpha_{m}^{\phi}(u)=\sup _{x^{*} \in B_{\left(\ell^{\phi}\right)^{*}} \cap\left(\left(\ell^{\phi}\right)^{*}\right)^{+}} \inf _{\operatorname{rep}(u)}\left(\sum_{i=1}^{\infty} x_{i}^{*}\left\|\sum_{j=1}^{n}\left|h_{j}\right|\right\| y_{j}\left\|_{Y}\right\|_{\ell^{\phi}}\right), \quad u \in L^{1}(\mu) \otimes Y .
$$

As in the case (1), this allows to prove that

$$
\alpha^{\phi}(u)=\left\|\left(\left\|u \chi_{A_{i}}\right\|_{L^{1}\left(\mu_{i}\right)}\right)_{i=1}^{\infty}\right\|_{\ell^{\phi}} .
$$


Example 3. Let us show now a different example regarding vector measures associated to classical operators. Consider the Lebesgue measure space $([0,1], \mathcal{B}, d x)$ Take the Volterra operator $V$ having values in $L^{1}[0,1]$, that is given by $V(f)(x):=$ $\int_{0}^{x} f(t) d t$ and consider the space of all the ( $\mu$-a.e. equal) measurable functions that satisfy that the Volterra operator is well-defined, that is, all the functions $f$ satisfying that $x \rightsquigarrow \int_{0}^{x} f(t) d t \in L^{1}[0,1]$. This is a Banach function space that can be represented as the space of integrable functions $L^{1}\left(m_{V}\right)$ with respect to the Volterra measure $m_{V}: \mathcal{B} \rightarrow L^{1}[0,1]$ given by $A \rightsquigarrow \int_{0}^{x} \chi_{A}(t) d t$ (see Example 3.26 in $\left[18\right.$, Ch.3]). For an element $h \in B_{\left(L^{1}[0,1]\right)^{*}}=B_{L^{\infty}[0,1]}$, we have that

$$
\left|\left\langle m_{V}, h\right\rangle\right|(A)=\int\left(\int_{0}^{x} \chi_{A}(t) d t\right)|h| d x, \quad A \in \mathcal{B} .
$$

Note that all of them are order bounded by

$$
\mu_{0}(A):=\left|\left\langle m_{V}, h\right\rangle\right|(A) \leq\left|\left\langle m_{V}, \chi_{\Omega}\right\rangle\right|(A)=\int_{0}^{1}\left(\int_{A \cap[0, x]} d t\right) d x, \quad A \in \mathcal{B}, x \in[0,1] .
$$

Let $Y$ be a Banach space. Let us give an explicit description of the space of vector valued Volterra functions $L^{1}\left(m_{V}, Y\right)$. By Theorem 1 , we have that

$$
L^{1}\left(m_{V}, Y\right)=L^{1}\left(m_{V}\right) \hat{\otimes}_{\alpha_{m_{V}}} Y,
$$

where the norm $\alpha_{m_{V}}$ is given for a simple tensor $u=\sum_{i=1}^{n} f_{i} \otimes y_{i}$ by

$$
\begin{aligned}
\alpha_{m_{V}}(u) & =\sup _{h \in B_{L^{\infty}[0,1]}} \inf _{\operatorname{rep}(u)}\left(\sum_{i=1}^{n}\left\|f_{j}\right\|_{L^{1}\left(\left|\left\langle m_{V}, h\right\rangle\right|\right)} \cdot\left\|y_{i}\right\|_{Y}\right) \\
& =\inf _{\operatorname{rep}(u)}\left(\sum_{i=1}^{n}\left\|f_{j}\right\|_{L^{1}\left(\mu_{0}\right)} \cdot\left\|y_{i}\right\|_{Y}\right)=\pi(u) .
\end{aligned}
$$

Therefore, we obtain a weighted $L^{1}$-space, and so the space can be identified with the projective tensor product of $L^{1}\left(\mu_{0}\right)$ and $Y$. That is, the Volterra space of vector valued functions $L^{1}\left(m_{V}, Y\right)$ coincides with the Bochner space $L^{1}\left(\mu_{0}, Y\right)$.

3.2. The dual space of $L^{1}(\nu, Y)$ of a positive vector measure $\nu$. Assume that $\nu$ is a positive vector measure with values in the Banach lattice $L$ and let $Y$ be a Banach space. Then we can obtain a good representation of the dual space of $L^{1}(\nu, Y)$ in terms of operators that factor through $L^{1}$-spaces of scalar positive finite measures. Consider the space $\mathcal{G}\left(L^{1}(\nu), Y^{*}\right)$ of all operators that satisfy the following property. If $T \in \mathcal{G}\left(L^{1}(\nu), Y^{*}\right)$ there is a functional $x^{*} \in L^{*}$ such that $T$ factors as

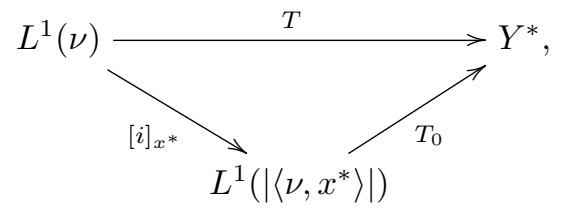

where $[i]_{x^{*}}$ is the inclusion/quotient map that sends each function in $L^{1}(\nu)$ to the equivalence class of $f$ with respect to the measure $\left|\left\langle\nu, x^{*}\right\rangle\right|$ (see [3]). Consider the 
norm given by the infimum of the norms of all suitable operators $\left\|T_{0}\right\|$ in this factorization, that clearly depends on $x^{*}$. In this section we show that $\left(L^{1}(\nu, Y)\right)^{*}$ can be represented isometrically as the spaces of all such operators, i.e. we will show that $\left(L^{1}(\nu, Y)\right)^{*}=\mathcal{G}\left(L^{1}(\nu), Y^{*}\right)$.

Recall that given a continuous bilinear form $\varphi: L^{1}(\nu) \times Y$ the linear map $T_{\varphi}$ : $L^{1}(\nu) \rightarrow Y^{*}$ given by $\left\langle y, T_{\varphi}(f)\right\rangle:=\varphi(f \otimes y), y \in Y, f \in L^{1}(\nu)$, is defined and continuous. Moreover, $\|\varphi\|=\left\|T_{\varphi}\right\|$. Conversely, if $T: L^{1}(\nu) \rightarrow Y^{*}$ is a continuous operator, the expression $\varphi_{T}(f, y):=\langle y, T(f)\rangle$, defines a continuous bilinear form as above with same norm than $T$, i.e. there is an isometric identification among continuous maps and bilinear forms.

Theorem 4. Let $\nu$ be an $L$-valued positive vector measure and let $\varphi$ be a continuous bilinear map $\varphi: L^{1}(\nu) \times Y \rightarrow \mathbb{R}$. The following statements are equivalent.

(i) $\varphi \in\left(L^{1}(\nu) \otimes_{\alpha_{\nu}} Y\right)^{*}$.

(ii) For all finite sets $f_{1}, \ldots f_{n} \in L^{1}(\nu)$ and $y_{1}, \ldots, y_{n} \in Y$,

$$
\sum_{i=1}^{n} \varphi\left(f_{i}, y_{i}\right) \leq\|\varphi\| \cdot\left\|\int \sum_{i=1}^{n}\right\| y_{i}\left\|\left|f_{i}\right| d \nu\right\|_{L}
$$

(iii) There is an element $x_{0}^{*} \in B_{L^{*}} \cap\left(L^{*}\right)^{+}$such that the operator $T_{\varphi}$ factors as

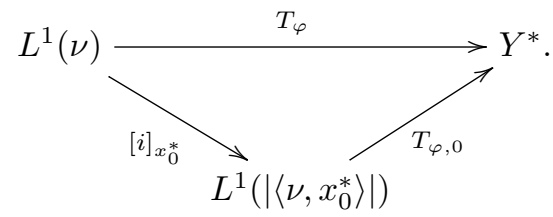

Moreover, $\|\varphi\|_{\left(L^{1}(\nu) \otimes_{\alpha_{\nu}} Y\right)^{*}}=\inf \left\|T_{\varphi, 0}\right\|$, where the infimum is computed over all suitable factorizations like the one above.

Proof. (i) $\Rightarrow$ (ii) Suppose that $\varphi \in\left(L^{1}(\nu, Y)\right)^{*}$. For each pair of finite sets $f_{1}, \ldots f_{n} \in$ $L^{1}(\nu)$ and $y_{1}, \ldots, y_{n} \in Y$, consider the tensor $\sum_{i=1}^{n} f_{i} \otimes y_{i}$. Then we have that

$$
\begin{gathered}
\sum_{i=1}^{n} \varphi\left(f_{i}, y_{i}\right)=\varphi\left(\sum_{i=1}^{n} f_{i} \otimes y_{i}\right) \\
\leq\|\varphi\| \alpha_{\nu}(u) \leq\|\varphi\| \sup _{x^{*} \in B_{L^{*}}}\left(\sum_{i=1}^{n}\left\|y_{i}\right\| \cdot\left\|f_{i}\right\|_{L^{1}\left(\left|\left\langle\nu, x^{*}\right\rangle\right|\right)}\right) .
\end{gathered}
$$

Since $\nu$ is a positive vector measure and using the fact that the functions $f_{i}$ are $\nu$-integrable, we have that the expression on the right hand side can also be written as

$$
\begin{gathered}
\sup _{x^{*} \in B_{L^{*}} \cap\left(L^{*}\right)^{+}}\left(\sum_{i=1}^{n}\left\|y_{i}\right\| \int\left|f_{i}\right| d\left\langle\nu, x^{*}\right\rangle\right)=\sup _{x^{*} \in B_{L^{*}} \cap\left(L^{*}\right)^{+}}\left\langle\sum_{i=1}^{n}\left\|y_{i}\right\| \int\left|f_{i}\right| d \nu, x^{*}\right\rangle \\
=\left\|\int \sum_{i=1}^{n}\right\| y_{i}\left\|\left|f_{i}\right| d \nu\right\| .
\end{gathered}
$$


The domination inequality at the beginning is then equivalent to

$$
\begin{gathered}
\sum_{i=1}^{n} \varphi\left(f_{i}, y_{i}\right) \leq\|\varphi\| \cdot\left\|\int\left(\sum_{i=1}^{n}\left\|y_{i}\right\|\left|f_{i}\right|\right) d \nu\right\| \\
=\|\varphi\| \cdot \sup _{x^{*} \in B_{L^{*}}} \int \sum_{i=1}^{n}\left\|y_{i}\right\|\left|f_{i}\right| d\left\langle\nu, x^{*}\right\rangle=\|\varphi\|\left\|\int \sum_{i=1}^{n}\right\| y_{i}\left\|\left|f_{i}\right| d \nu\right\|
\end{gathered}
$$

for all finite sets $f_{1}, \ldots f_{n} \in L^{1}(\nu)$ and $y_{1}, \ldots, y_{n} \in Y$.

(ii) $\Rightarrow$ (iii) We apply a standard separation argument based on Ky Fan's Lemma - or on the Hahn-Banach Theorem, see for example the arguments in [18, Lemma 6.13] or [4, Theorem 6] - to obtain that there is an element $x_{0}^{*} \in B_{L^{*}} \cap\left(L^{*}\right)^{+}$such that

$$
\sum_{i=1}^{n}\left\langle T_{\varphi}\left(f_{i}\right), y_{i}\right\rangle \leq\|\varphi\| \int \sum_{i=1}^{n}\left\|y_{i}\right\|\left|f_{i}\right| d\left\langle\nu, x_{0}^{*}\right\rangle .
$$

Consequently, for each $f \in L^{1}(\nu)$ and $y \in Y$, we have that

$$
\left\langle T_{\varphi}(f), y\right\rangle \leq\|\varphi\|\|y\| \cdot \int|f| d\left\langle\nu, x_{0}^{*}\right\rangle .
$$

This clearly implies the factorization for $T_{\varphi, 0}:=T$ in $L^{1}(\nu)$, since $[i]_{x_{0}^{*}}\left(L^{1}(\nu)\right)$ is dense in $L^{1}\left(\left\langle\nu, x_{0}^{*}\right\rangle\right)$. The inequality $\left\|T_{\varphi, 0}\right\| \leq\|\varphi\|$ is also obtained, and so the proof is done.

(iii) $\Rightarrow$ (i) Assume that there is a factorization as the one given for $T_{\varphi}$. Let us show that it defines a continuous functional on $L^{1}(\nu, L)=L^{1}(\nu) \hat{\otimes}_{\alpha_{\nu}} Y$. For each $u=\sum_{i=1}^{n} f_{i} \otimes y_{i} \in L^{1} \otimes_{\alpha_{\nu}} Y$, we have that

$$
\begin{aligned}
\left|\left\langle u, T_{\varphi}\right\rangle\right| & =\left|\sum_{i=1}^{n}\left\langle y_{i}, T_{\varphi}\left(f_{i}\right)\right\rangle\right|=\left|\sum_{i=1}^{n}\left\langle y_{i}, T_{\varphi, 0}\left([i]_{x_{0}^{*}}\left(f_{i}\right)\right)\right\rangle\right| \\
& \leq\left\|T_{\varphi, 0}\right\| \sum_{i=1}^{n}\left\|y_{i}\right\| \cdot\left\|f_{i}\right\|_{L^{1}\left(\left|\left\langle\nu, x_{0}^{*}\right\rangle\right|\right)} .
\end{aligned}
$$

Note that these computations work for any representation of $u$, and so

$$
\left|\left\langle u, T_{\varphi}\right\rangle\right| \leq\left\|T_{\varphi, 0}\right\| \inf _{\operatorname{rep}(u)} \sum_{i=1}^{n}\left\|y_{i}\right\| \cdot\left\|f_{i}\right\|_{L^{1}\left(\left|\left\langle\nu, x_{0}^{*}\right\rangle\right|\right)},
$$

which implies that

$$
\left|\left\langle u, T_{\varphi}\right\rangle\right| \leq\left\|T_{\varphi, 0}\right\| \sup _{x^{*} \in B_{L^{*}}} \inf _{\operatorname{rep}(u)} \sum_{i=1}^{n}\left\|y_{i}\right\| \cdot\left\|f_{i}\right\|_{L^{1}\left(\left|\left\langle\nu, x^{*}\right\rangle\right|\right)} .
$$

Hence $T_{\varphi} \in\left(L^{1} \hat{\otimes}_{\alpha_{\nu}} Y\right)^{*}$ and $\left\|T_{\varphi}\right\|_{\left(L^{1} \hat{\otimes}_{\alpha_{\nu}} Y\right)^{*}} \leq\left\|T_{\varphi, 0}\right\|$.

The relations between the norms that have been shown in (ii) $\Rightarrow$ (iii) and (iii) $\Rightarrow$ (i) prove also the coincidence of the norms.

This result leads to the following one, just taking into account the one to one relation among bilinear forms and continuous linear maps. 
Corollary 5. Let $\nu: \Sigma \rightarrow L$ be a positive vector measure. Then

$$
\left(L^{1}(\nu, Y)\right)^{*}=\mathcal{G}\left(L^{1}(\nu), Y^{*}\right)
$$

isometrically.

Remark 6. A relevant fact that is shown in the proof of Theorem 4 is that each element of the dual space $\left(L^{1}(\nu) \otimes_{\alpha_{\nu}} Y\right)^{*}$ satisfy the inequality

$$
|\varphi(f, y)| \leq\|\varphi\| \cdot\|y\| \cdot \int|f| d\left\langle\nu, x_{0}^{*}\right\rangle,
$$

for a certain positive norm one functional $x_{0}^{*}$ depending only on $\varphi$, and in fact this relation characterizes the elements of the dual space. This kind of inequalities are closely related to the so called Radon-Nikodým Theorem for vector valued measures that are studied in [4]: operators factoring through the $L^{1}$-spaces of a scalar measure associated to the vector measure has been intensively studied in this paper.

Example 7. Consider the space $Z:=\bigoplus_{i=1, p}^{\infty} L^{1}\left(\mu_{i}\right)$ given in Example $2,1<p<\infty$. Let $Y$ be a Banach space such that $Y^{*}$ has the Radon-Nikodým property. We know that the Köthe-Bochner space $Z(\eta, Y)$ - where $\eta$ was defined in Example 2-, can be identified with

$$
L^{1}(m, Y)=L^{1}(m) \hat{\otimes}_{\alpha_{m}} Y,
$$

for the vector measure $m$ defined in Example 2. On the other hand, using for example Theorem 1 in $[10$, Ch.IV], we can easily prove that

$$
(Z(\mu, Y))^{*}=\bigoplus_{i=1, p^{\prime}}^{\infty}\left(L^{1}\left(\mu_{i}, Y\right)\right)^{*}=\bigoplus_{i=1, p^{\prime}}^{\infty} L^{\infty}\left(\mu_{i}, Y^{*}\right) .
$$

Theorem 4 provides an alternate description of the dual space; note that the vector measure $m$ used in this example is positive. If $\left(\beta_{i}\right) \in B_{\ell^{p^{\prime}}}$, the measure $\left\langle m,\left(\beta_{i}\right)\right\rangle$ is given by

$$
\left\langle m,\left(\beta_{i}\right)\right\rangle(A)=\sum_{i=1}^{\infty} \beta_{i} \mu\left(A \cap A_{i}\right), \quad A \in \Sigma .
$$

Consider the bilinear maps $\varphi: L^{1}(m) \times Y \rightarrow \mathbb{R}$ and the operators $T_{\varphi}$ that are defined as $T_{\varphi}(f)(y)=\varphi(f \otimes y), f \in L^{1}(m), y \in Y$. The dual space can be described as the space of all the bilinear maps $\varphi$ with associated operators $T_{\varphi}: L^{1}(m) \rightarrow Y^{*}$ for which there is an element $0 \leq \beta_{0}=\left(\beta_{i}\right)_{i=1}^{\infty} \in B_{\ell^{p^{\prime}}} \cap\left(\ell^{p^{\prime}}\right)^{+}$such that $T_{\varphi}$ factors as

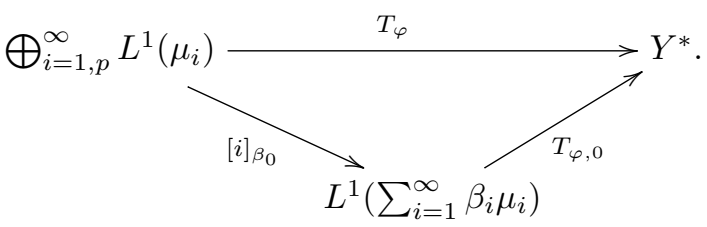

The norm of such a $\varphi$ as an element of the dual space of $L^{1}(m, Y)$ is given by the infimum of $\left\|[i]_{\beta_{0}}\right\| \cdot\left\|T_{\varphi, 0}\right\|$ for all suitable factorizations like that. 
We have required to the space $Y$ to have the Radon-Nikodým property in Example 7 for having the standard representation of the dual space of $Z(\eta, Y)$, but note that this fact does not affect the factorization property that characterizes the representation of the dual given by Theorem 4. Let us write now a direct consequence of our results when this requirement is added. It regards the classic topic of representation of vector measures and operators on spaces whose dual spaces have the Radon-Nikodým property (Asplund spaces).

Corollary 8. Assume that $\nu$ is an L-valued positive vector measure and $Y^{*}$ has the Radon-Nikodym property. Let $\varphi \in\left(L^{1}(\nu) \otimes_{\alpha_{\nu}} Y\right)^{*}$. Then there are a positive element $x_{0}^{*} \in\left(L^{*}\right)^{+}$and a function $g_{\varphi} \in L^{\infty}\left(\left\langle\nu, x_{0}^{*}\right\rangle, Y^{*}\right)$ such that

$$
T_{\varphi}(f)=\int f g_{\varphi} d\left\langle\nu, x_{0}^{*}\right\rangle
$$

for all $f \in L^{1}(\nu)$.

Proof. This is a straightforward consequence of Theorem 4, Corollary 5 and [10, Theorem 5].

More concrete representations of the dual spaces are possible under some requirements on the representation of the dual space of the space $L^{1}(\nu)$. Since the space is order continuous, $\left(L^{1}(m)\right)^{*}$ coincides with its Köthe dual $\left(L^{1}(m)\right)^{\prime}$, that is a Banach function space (see [17, p.28]). Suppose that $B_{\left(L^{1}(\nu)\right)^{\prime}}=\Gamma \cdot B_{L^{\infty}(\nu)}$, where $\Gamma:=\left\{\varphi \in\left(L^{1}(\nu)\right)^{\prime}: \varphi=d\left|\left\langle\nu, x^{*}\right\rangle\right| / d \eta, x^{*} \in B_{X^{*}}\right\}$ and $\eta$ is a fixed Rybakov measure for $\nu$.

Remark 9. Other useful formulae can be obtained by applying this result. Suppose that $Y^{*}$ has the Radon-Nikodým property and $\nu$ is positive. Fix $\varphi \in\left(L^{1}(\nu), X^{*}\right)$ and consider the operator $T_{\varphi}$. Then there are an element $0 \leq x_{0}^{*} \in B_{X^{*}}$, and an operator $T_{\varphi, 0}$ such that $T_{\varphi}=T_{\varphi, 0} \circ[i]_{x_{0}^{*}}$. Write $g$ for the function $g \in L^{\infty}\left(\left\langle\nu, x_{0}^{*}\right\rangle, Y^{*}\right)$ that is the Radon-Nikodým derivative of $T_{\varphi, 0}: L^{1}\left(\left\langle\nu, x_{0}^{*}\right\rangle\right) \rightarrow Y^{*}$. If $y \in Y$ and $f \in L^{1}(\nu)$, we have that

$$
\begin{aligned}
\varphi(f & \otimes y)=\left\langle y, T_{\varphi, 0} \circ[i]_{x_{0}^{*}}(f)\right\rangle=\int T_{\varphi, 0}^{*}(y) f d\left\langle\nu, x_{0}^{*}\right\rangle \\
& =\int\langle g, y\rangle f d\left\langle\nu, x_{0}^{*}\right\rangle=\left\langle\int\langle g, y\rangle f d \nu, x_{0}^{*}\right\rangle .
\end{aligned}
$$

Consequently, we obtain the following formula for the action of $\varphi$ on a tensor $t=\sum_{i=1}^{n} f_{i} \otimes y_{i} \in L^{1}(m) \otimes Y:$

$$
\varphi(t)=\left\langle\int\left(\sum_{i=1}^{n}\left\langle g, y_{i}\right\rangle f_{i}\right) d \nu, x_{0}^{*}\right\rangle .
$$




\section{Applications}

In this section we show two particular applications of the representation results that have been shown before. The first one shows how may them improve the knowledge on the description of the dual of abstract Köthe-Bochner spaces without involving vector measures. The second one comes back to the original problem: the tensor representation of spaces of Bochner $p$-integrable functions. We assume that $(\Omega, \Sigma, \mu)$ is a purely non-atomic finite measure space.

\subsection{Concrete representations of the dual of Köthe-Bochner spaces. In}

this section we provide some applications of the representation of the dual space given in the previous one. Consider an order continuous Banach lattice $L$ with order continuous norm and a weak unit. Then it can be represented as an $L^{1}$ of a positive vector measure $\nu$ (see [18, Proposition 3.9] and the references therein), i.e. there is an isometric and order identification $L=L^{1}(\nu)$. In fact, in case we have $L$ represented already as a space of integrable functions $X(\mu)$ over a finite measure $\mu$-using the Kakutani representation theorem, see for instance [17, Theorem 1.b.14] - the vector measure $\vartheta$ that provides the isometric equality $L^{1}(\vartheta)=X(\mu)$ is just given by $\vartheta(A):=\chi_{A} \in X(\mu)$, and the measurable target space $(\Omega, \Sigma)$ is the same for $\mu$ and $\vartheta$.

The results previously obtained in this paper show more concrete representations in this case. Clearly, each positive scalar measure $\left\langle\vartheta, x^{*}\right\rangle, x^{*} \in X(\mu)^{*}=X(\mu)^{\prime}$ associated to $\nu$ is given by an integral as

$$
\left\langle\vartheta, x^{*}\right\rangle(A)=\int_{A} h d \mu, \quad A \in \Sigma
$$

for a certain function $h \in X(\mu)^{\prime}$. Using this, we can give the following representation of the dual of the Köthe-Bochner space $X(\mu, Y)=L^{1}(\vartheta, Y)$.

Corollary 10. Let $X(\mu)$ be an order continuous Banach function space over the finite measure $\mu$. Let $\varphi$ be a continuous bilinear form on $X(\mu) \times Y$. The following statements are equivalent.

(i) $\varphi \in(X(\mu, Y))^{*}$.

(ii) There is an integrable function $h \in X(\mu)^{\prime}$ such that the diagram



commutes.

In this case, $\|\varphi\|_{(X(\mu, Y))^{*}}=\inf \left\|T_{h}\right\|_{L\left(L^{1}(h d \mu), Y^{*}\right)}$, where the infimum is computed over all $h$ in the unit sphere of $X(\mu)^{\prime}$. 
Moreover, using Corollary 8 we obtain the following concrete representations of the elements of the dual space of the Köthe-Bochner space $X(\mu, Y)$.

Corollary 11. Let $X(\mu)$ be an order continuous Banach function space over the finite measure $\mu$. Suppose that $Y^{*}$ has the Radon-Nikodym property. Let $\varphi \in$ $(X(\mu, Y))^{*}$. Then there are a positive element $h \in X(\mu)^{\prime}$ and a function $g_{\varphi} \in$ $L^{\infty}\left(h d \mu, Y^{*}\right)$ such that

$$
T_{\varphi}(f)=\int f g_{\varphi} h d \mu
$$

for all $f \in X(\mu)$.

4.2. The natural norm on the tensor product $L^{p}(\mu) \otimes Y$ revisited. Let $1 \leq$ $p<\infty$ and write $p^{\prime}$ for the (extended) real number satisfying that $1 / p+1 / p^{\prime}=1$. Recall that the norm $\Delta_{p}$ for the tensor product $L^{p}(\mu) \otimes Y$ that provides the isometry $L^{p}(\mu) \hat{\otimes}_{\Delta_{p}} Y=L^{p}(\mu, Y)$ (see [8, Chapter 7 and Section 15.10]) is not a truly tensor norm since it does not satisfy the mapping property required for this to hold (see [8, Section 7.3]). However, - as we said at the beginning of the paper and is actually its original motivation - we have that $L^{1}(\mu) \hat{\otimes}_{\pi} Y=L^{1}(\mu, Y)$, i.e. $\Delta_{1}=\pi$. This is our norm $\alpha_{p}$ for the extreme case $p=1$-and so $p^{\prime}=\infty$ - Let us show that the rest of the norms of this family can be represented in a $\pi$-norm fashion, instead of the usual representation of the norms $\Delta_{p}$ as the Bochner $p$-norm of the vector valued function defined by a tensor.

In this case, our results provide an alternate representation of the Köthe-Bochner spaces $L^{p}(\mu, Y)$ by means of tensor products. If $(\Omega, \Sigma, \mu)$ is a finite measure space, we can consider as in the previous section the representation of $L^{p}(\mu)$ as $L^{1}(\vartheta)$ for the vector measure $\vartheta$ given by $\vartheta(A):=\chi_{A} \in L^{p}(\mu)$. Let us write $\alpha_{p}$ for the norm $\alpha_{\nu}$ in this case. It is defined as follows. If $u=\sum_{i=1}^{n} h_{i} \otimes y_{i} \in L^{p}(\mu) \otimes Y$, we find that

$$
\begin{aligned}
\alpha_{p}(u) & :=\sup _{g \in B_{L^{p^{\prime}(\mu)}}} \inf _{\operatorname{rep}(u)}\left(\sum_{i=1}^{n}\left(\int\left|h_{i}\right||g| d \mu\right) \cdot\left\|y_{i}\right\|_{Y}\right) \\
= & \sup _{g \in B_{L^{p^{\prime}(\mu)}}} \inf _{\operatorname{rep}(u)}\left(\int\left(\sum_{i=1}^{n}\left|h_{i}\right|\left\|y_{i}\right\|_{Y}\right)|g| d \mu\right) \\
= & \sup _{g \in B_{L^{p^{\prime}(\mu)}}} \inf _{\operatorname{rep}(u)}\left\|\sum_{i=1}^{n}\left|h_{i}\right|\right\| y_{i}\left\|_{Y}\right\|_{L^{1}(|g| d \mu)},
\end{aligned}
$$

where $\operatorname{rep}(u)$ denotes the set of all the representations of $u$ as the one given.

Regarding the dual space, recall that under the assumption for $Y^{*}$ to have the Radon-Nikodým property, we obtain that $\left(L^{p}(\mu, Y)\right)^{*}=L^{p^{\prime}}\left(\mu, Y^{*}\right)$ (see for example Theorem 1 in [10, Ch.IV]). Note that an application of Theorem 4 gives that a linear functional $\phi: L^{p}(\mu, Y) \rightarrow \mathbb{R}$ belongs to $\left(L^{p}(\mu, Y)\right)^{*}$ if and only if there is a constant 
$K \geq 0$ such that for all finite sets $f_{1}, \ldots f_{n} \in L^{p}(\mu)$ and $y_{1}, \ldots, y_{n} \in Y$,

$$
\phi\left(\sum_{i=1}^{n} f_{i} \cdot y_{i}\right) \leq K\left(\int\left(\sum_{i=1}^{n}\left\|y_{i}\right\|\left|f_{i}\right|\right)^{p} d \mu\right)^{1 / p},
$$

or, equivalently, a factorization scheme for the operator $T_{\varphi}$ as the following is given, for $\varphi(f, y):=\phi(f \cdot y), f \in L^{p}(\mu), y \in Y, \phi \in\left(L^{p}(\mu, Y)\right)^{*}:$

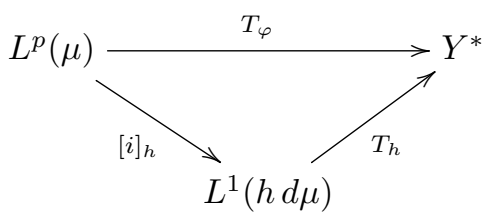

for some $h \in L^{p^{\prime}}(\mu)$. No Radon-Nikodým property for the spaces involved is needed for these characterizations. However, if $Y^{*}$ has the Radon-Nikodým property, we obtain that the unique operators $T_{\varphi}$ that factor as above for a fixed bilinear map $\varphi$ associated to a linear functional $\phi$ are the ones for which there is a function $g \in L^{p^{\prime}}\left(\mu, Y^{*}\right)$ such that

$\left\langle y, T_{\varphi}(f)\right\rangle=\int f(w)\langle y, g(w)\rangle d \mu(w)=\left\langle y, \int f(w) g(w) d \mu(w)\right\rangle, \quad f \in L^{p}(\mu), y \in Y$,

that is,

$$
T_{\varphi}(f)=\int f(w) g(w) d \mu(w), \quad f \in L^{p}(\mu)
$$

\section{REFERENCES}

[1] S. Bochner, Integration von Funktionen, deren Werte die Elemente eines Vectorraumes sind, Fundamenta Mathematicae 20, (1933) 262-276.

[2] J. M. Calabuig, O. Delgado, M. A. Juan and E. A. Sánchez Pérez, On the Banach lattice structure of $L_{w}^{1}$ of a vector measure on a $\delta$-ring. Collect. Math. 65 (2014), 6567-85.

[3] J. M. Calabuig, O. Delgado and E. A. Sánchez Pérez, Factorizing operators on Banach function spaces through spaces of multiplication operators, J. Math. Anal. Appl. 364,1 (2010), 88-103.

[4] J. M. Calabuig, P. Gregori and E. A. Sánchez Pérez, Radon-Nikodým derivatives for vector measures belonging to Köthe function spaces, J. Math. Anal. Appl. 348 (2008), 469-479.

[5] J. Cerdà, H. Hudzik and M. Mastyło, Geometric properties of Köthe-Bochner spaces. Math. Proc. Cambridge Philos. Soc. 120,3 (1996), 521-533.

[6] N. D. Chakraborty and S. Basu, Spaces of p-tensor integrable functions and related Banach space properties, Real Anal. Exchange 34 (2008/2009), 87-104.

[7] N. D. Chakraborty and S. Basu, Integration of vector-valued functions with respect to vector measures defined on $\delta$-rings, Illinois J. Math. 55, 2 (2011) 495-508.

[8] A. Defant and K. Floret, Tensor norms and operator ideals, North-Holland, Amsterdam, 1993.

[9] O. Delgado and M. A. Juan, Representation of Banach lattices as $L_{w}^{1}$ spaces of a vector measure defined on a $\delta$-ring. Bull. Belgian Math. Soc. 19 (2012) 239-256.

[10] J. Diestel and J.J. Uhl, Vector measures, Am. Math. Soc., Providence, 1977.

[11] I. Dobrakov, On integration in Banach spaces, VII, Czechoslovak Math. J. 38 (1988), 434449. 
14 J.M. CALABUiG, E. JIMÉNEZ FERNÁNDEZ, M.A. JUAN AND E.A. SÁNCHEZ PÉREZ

[12] L.M.García-Raffi and B.Jefferies, An application of bilinear integration to quantum scattering, J. Math. Anal. Appl. 415 (2014), 394-421.

[13] P. Gregori Huerta, Espacios de medidas vectoriales, Thesis, Universidad de Valencia, ISBN:8437060591, 2005.

[14] B. Jefferies and S. Okada, Bilinear integration in tensor products, Rocky Mountain J. Math. 28 (1998), 517-545.

[15] D. R. Lewis, On integrability and summability in vector spaces, Illinois J. Math. 16 (1972) 294-307.

[16] P.-K. Lin, Köthe-Bochner function spaces, Birkhauser, Boston, 2004.

[17] J. Lindenstrauss and L. Tzafriri, Classical Banach Spaces II, Springer, Berlin, 1979.

[18] S. Okada, W. J. Ricker and E. A. Sánchez Pérez, Optimal Domains and Integral Extensions of Operators acting in Function Spaces, Operator Theory Advances and Applications, vol.180, Birkhäuser, Basel, 2008.

[19] R. Pallu de La Barriére, Integration of vector functions with respect to vector measures, Studia Univ. Babes-Bolyai Math. 43 (1998), 55-93.

[20] J. Rodríguez, On integration of vector functions with respect to vector measures, Czechoslovak Math. J. 56 (2006), 805-825.

J.M. Calabuig and E. A. Sánchez Pérez, Instituto Universitario de Matemática Pura y Aplicada, Universidad Politécnica de Valencia, Valencia, Spain

E-mail address: jmcalabu@mat.upv.es, easancpe@mat.upv.es

M. A. Juan, Universidad Católica de Valencia, Valencia, Spain

E-mail address: majuabl1@mat.upv.es

E. Jiménez Fernández, Departamento de Economía Aplicada, Universitat Jaume I, CAstellón, Spain

E-mail address: jimeneze@uji.es 